Introduction Selected young febrile infants with urinary tract infection (UTI) and lower risk for associated bacteremia can be managed as outpatients. Different biomarkers are useful in the management of febrile young infants but their role in this specific population has not been clarified.

Objective To analyze the relationship between blood biomarkers (white blood cell count -WBC-, absolute neutrophil count -ANC-, procalcitonin -PCT- and C-reactive protein -CRP) and the risk of bacteremia in infants under three months with febrile UTI.

Patients and Methods Prospective multicentric study developed in 29 Spanish Paediatric Emergency Departments members of the RISEUP-SPERG (Spanish Pediatric Emergency Research Group), including febrile infants less than three months old diagnosed with UTI between October-2011 and March-2012.

Results We included 715 infants, being 151 (21.1\%) diagnosed with UTI. Forty-five (28.6\%) were excluded because not having obtained CRP or PCT. Finally, 106 febrile infants under three months diagnosed with UTI were included (5-4.7\%- with associated bacteremia). Values of WBC and ANC were similar in infants with and without bacteremia (14116 leucocytes $/ \mathrm{mm}^{3}$, CI 95\% 11178-17053 vs 15630 , CI 95\% 14221-17039; and 8912 neutrophils $/ \mathrm{mm}^{3}$, CI 95\% 4865-12960 vs 8351, CI 95\% 7327-9375; respectively). Values of CRP and PCT were significantly lower in patients without bacteremia when compared with those with bacteremia $(107.7 \mathrm{mg} / \mathrm{L}, \mathrm{CI}$ 95\% 60.1-155.3; vs 48.8, CI95\% 37.9-59.7; and 26.3, CI 95\% 6.845.9 vs 2.6 , CI 95\% $1.2-4.0$ )

Conclusion Procalcitonin and C-reactive protein identify better than classical biomarkers young febrile infants with UTI at higher risk for bacteremia.

\section{REDUCED CONTAMINATION OF BLOOD CULTURES DRAWN IN THE ED. IMPACT OF THE IMPLEMENTATION OF AN EXTRACTION PROTOCOL}

doi:10.1136/archdischild-2012-302724.1476

'R Velasco, ' JL Fernandez, 'N Campo, 'S Puente, ${ }^{2} \mathrm{M}$ Dominguez-Gil, 'M Sanchez,

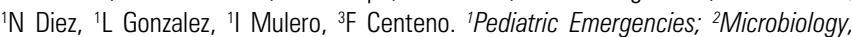
${ }^{3}$ Pediatrics, Hospital Rio Hortega, Valladolid, Spain

Introduction Blood culture is the gold standard for the diagnosis of bacteremia. Clinical guidelines consider adequate a contamination rate of less than 3\%. Between 2009 and 2010 in our service this rate was $12^{\prime} 56 \%$. Therefore, in February-2011 we started a protocol for blood cultures according to the Scientific Evidence.

Objectives Analyze the impact of implementing a protocol for the extraction of hemocultures on contamination rates.

Methods Retrospective study cohort: Pre-implantation group (A): Patients whom a blood culture was taken from in our Pediatric Emergency Department between March 2009 and April 2010 and post-implantation group (B): Patients with blood culture between April 2011 and March 2012.

Results In the group A 1098 blood cultures were obtained (4'08\% of 26874 patients attended) and in group B 535 (2'87\% of 18628 patients, $\mathrm{p}=0.0001)$. A true pathogen was isolated in 11 samples in cohort A $\left(1^{\prime} 00 \%\right.$, CI95\% $0.41-1.59 ; 0.04 \%$ of the population attended from CI $95 \% 0.02$ to 0.06$)$ and in 9 of the group B (1'68\%; CI $95 \% 0.59-2.77 ; 0$; $048 \%$ of the population attended CI $95 \% 0.02$ to 0.08$)$.

In the group A germs considered contaminants grew in 138 blood cultures $\left(12^{\prime} 56 \%\right.$, CI $\left.95 \% 10.6-14.52\right)$ and in 34 in the B (6'35\%, C195\%: 4.28-8.42).

Conclusion The development of a protocol for blood cultures extraction technique according to the Scientific Evidence is useful to reduce sample contamination, but it didn't reach to the standards of quality. Technique protocolization was associated with a significant decrease in number of blood cultures drawn.

\section{PROGNOSTIC VALUE OF INITIAL ARTERIAL LACTATE LEVELS OF ACUTE CARBON MONOXIDE POISONING IN CHILDREN}

doi:10.1136/archdischild-2012-302724.1477

S Sahin. Eskisehir Osmangazi University, Eskisehir, Turkey

Aim The present study was aimed to compare the prognostic values of lactate and carboxyhemoglobin levels on clinical neurological and cardiac involvement in children with carbon monoxide poisoning.

Methods Totally 77 children aged to 1 month and 17 years who referred to Paediatric Emergency Department (PED) between 1 April 2009 and 30 April 2011 and diagnosed with carbon monoxide intoxication were assessed for age, gender, symptoms, clinical findings, blood gas lactate levels, carboxyhemoglobin levels, morbidity and mortality.

Results There was a significant positive correlation between initial lactate levels in blood gas analysis and early and late complications, neurological and cardiac involvements and morbidity compared to carboxyhemoglobin levels.

Discussion The initial lactate levels have higher prognostic value for cardiac involvement and late neurological sequelae compared to carboxyhemoglobin and it may be helpful for treatment.

\begin{tabular}{l}
\hline 1478 ATTENDANCES AT THE CHILDREN'S EMERGENCY \\
DEPARTMENT: AN AUDIT OF TWO YEARS OF ACTIVITY \\
AT A NEW, DEDICATED PAEDIATRIC EMERGENCY \\
DEPARTMENT
\end{tabular}

doi:10.1136/archdischild-2012-302724.1478

1,2R Isba, ${ }^{2} \mathrm{M}$ Gibb, 'AG Rowland. 'Emergency Department, North Manchester General Hospital, Manchester; ${ }^{2}$ Medical School, Lancaster University, Lancaster, UK

Aim The aim of this audit was to explore patterns of attendance at a new, dedicated, audio-visually separate paediatric emergency department (PED) within the ED of a hospital in the North West of England. The PED was opened in June 2009 following the major restructuring of paediatric services in Manchester.

Methods This was a retrospective audit of paediatric attendances (those aged < 16 years) to the ED of North Manchester General Hospital between June 2009 and June 2011.

Results During the first year there were 26073 attendances to the PED, while in the second there were 24119 (just over a quarter of all attendances to the ED). More than half of all attendees were in the pre-school age group. There were low levels of attendance overnight, and numbers of attendees peaked around $6 \mathrm{pm}$. Mondays were the busiest days (mean attendees 75.4) and Fridays the quietest (mean attendees 64.6). Whilst the average length of stay was just over two hours, this varied depending on the time of arrival. Approximately one in six children was referred to an inpatient specialty for further assessment.

Conclusion These results have implications for those managing EDs that care for children. Knowledge of attendance patterns may enable managers to better plan staffing levels and deployment within an ED, and there are wider implications for the hospital as a whole. By gaining a better understanding of the patterns of attendance it may be possible to predict future patterns (e.g. via mathematical modelling), and to plan resources accordingly.

\section{REFERRAL OF CHILDREN FROM A UK DISTRICT GENERAL HOSPITAL EMERGENCY DEPARTMENT TO PRIMARY CARE GENERAL PRACTITIONERS}

doi:10.1136/archdischild-2012-302724.1479

${ }^{1} \mathrm{AG}$ Rowland, ${ }^{1,2} \mathrm{R}$ Isba. 'Emergency Department, North Manchester General Hospital, Manchester; ${ }^{2}$ Medical School, Lancaster University, Lancaster, UK 